\title{
Variables and Applications on Dry Magnetic Separator
}

\author{
Qin Xing Zong, Luo Zhen Fu*, Lv Bo \\ School of Chemical Engineering and Technology, China University of Mining and Technology, Xuzhou, 221116, China.
}

\begin{abstract}
Magnetic separation is an indispensable part of magnetic separation, and the dry magnetic separator can be selected under the condition of water shortage in China to ensure that our country can also be selected under the conditions of lack of some resources. The magnetic separator plays a role in improving the grade of ore, purifying solid and liquid materials, and recycling waste. With the application and development of magnetic separation technology, magnetic separation equipment is constantly updating and replacing, and dry magnetic separation has experienced remarkable technological progress over the past twenty years. There are many new ideas and techniques applied in magnetic separators. So far, dry magnetic separators have developed many different applications for mineral and coal processing, for induction roller magnetic separators for chromite. Cross-belt magnetic separator for removing harmful magnetic particles and paramagnetic particles .The lifting roller magnetic separator is used in the heavy mineral industry to separate garnet from monazite and rutile . Rare earth drum magnetic separator for fine feed dry magnetic separation sorting process and rare earth roller magnetic separator for zircon and rutile in heavy mineral sand industry. These magnetic separators have different applications, and the dry magnetic separator is also moving toward large-scale and easy-to-manufacture.
\end{abstract}

\section{INTRODUCTION}

In the past two or three decades, magnetic separators have undergone unusual advances, and now there are different magnetic separator designs that treat minerals with other standards on a modern basis. According to the different sorting principle of magnetic separator, it can be divided into cross-belt magnetic separator (CBMS), induction roller magnetic separator (IMRS), lift roller magnetic separator (LRMS), permanent magnet roller magnetic separator (RERMS). ), and Rare earth drum magnetic separator (RED). Magnetic separators are very useful for separating coarse particles, where the wet process requires subsequent processing. The dry separation process has many advantages and disadvantages compared to the wet process. In some tests, dry sorting is superior to wet processes such as water consumption, reuse of recycled water, management of tailings ponds, and so on.

There are different methods for classifying magnetic separators, but grouping of these units based on magnetic field strength can be divided into: (1) Dry electromagnetic magnetic separator, e.g., Induction roller magnetic separator, Lift roller magnetic separator, and Cross belt magnetic separator; (2) Dry permanent magnet magnetic separator. e.g., Rare earth roller magnetic separator and Rare earth drum magnetic separator

\section{Dry Electromagnetic Magnetic Separator}

\subsection{Induction Roller Magnetic Separator}

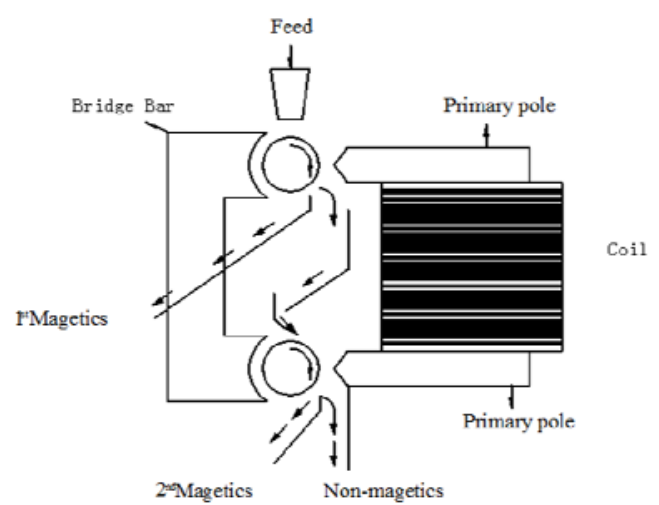

Figure. 1. Schematic diagram of particle separation in a twostage induction roll magnetic separator

The induction roller magnetic separator is a simple device consisting of a rotating laminated roll formed of a magnetizable and non-magnetic magnetic disk. The rolls consist of alternate lamina of permeable and impermeable material and the roll onto which the ore is fed is in a controlled thin stream by a vibratory feeder to the top roll. As the roll revolves, the material passes through a narrow gap between the pole of the magnet and the roll ${ }^{[1-2]}$. Schematic diagram of particle separation in an induction roll magnetic separator in two stages is depicted in Figure 1. When the magnetic particles are attracted to the roller, a non-magnetic particles are discharged from the roll, whereas when they enter the region outside the magnet will separate into another

\footnotetext{
* Corresponding author: zfluo@cumt.edu.cn
} 
chute. Before ferromagnetic material to induce the magnetic roll separator, to separate the ferromagnetic material should be removed. Roll steel laminate consisting of phosphate compressed together in the composition nonmagnetic stainless steel shaft. Laminating by using two different sizes of different outer diameters platen roller and the jagged sawtooth profile and gradient magnetic field intensity can be increased to the desired [1]. IRMS parameters affect separation field strength (applying a current gap between the rotor and the magnetic poles), feed rate, roll speed, the dispenser location. Other feed characteristics such as mineral surface conditions, feed particle size distribution, and density distribution vary with magnetic susceptibility. In addition, the moisture content of feed and slime content will determine the degree of separation. [2][4-6]

\subsubsection{Variables}

The variables affecting the sorting performance of the induction roller magnetic separator are divided into design variables and operational variables. The pole shape and rotor diameter are the main design variables, and the pole pitch, rotor speed, applied current, feed rate, and split position are the primary operational variables. In addition to these parameters, the characteristics of the feedstock, i.e. the particle size, the mineral magnetic susceptibility, density and shape of the particles, also is essential in the induction of separating the magnetic roll separator. The mineral machine specific conditions being treated in addition to the physical properties (i.e., magnetic susceptibility, density and size) of the particles is also affected by an external force. In addition, some of the effects of important variables discussed previously mentioned in the discussion section.

Sunil Kumar Tripathy determines the effect of test variables on grade by changing variables during mineral sorting experiments such as hematite., the effect of optimizing the magnetic field strength, roll speed and the feed rate of the three process variables, the modeling and design Box-Behnken response surface method and enthalpy concentrated hematite. Packages quadratic programming (Matlab 7.1) is calculated optimization to get the best grade and recovery of hematite and impact on the level of the experimental data observed variables optimization. ${ }^{[14]}$

i. Effect of variables on Fe content in magnetic products

Through the optimization of the test results ${ }^{[14]}$, the effects of magnetic field strength, rotor speed and feed rate on the $\mathrm{Fe}$ content of the magnetic product were observed. It is observed that higher grade is obtained at higher level of magnetic field intensity and intermediate level of feed rate. Also note that the rotor speed controls the particle trajectory which influences the selectivity further to increase the Fe content. This may be due to comparison with the silicate-containing gangue minerals, a higher value of the net force acting on the hematite particles. It was observed at a higher magnetic field strength and the rotor speed level lower case lower Fe content of the magnetic product, and demonstrates the effect of different rotor speeds mineral particles trajectory. Therefore, it can be inferred that at the central level of the rotor speed, as the strength of the magnetic field increases, the Fe content of the magnetic product increases. Similarly, small changes in the magnetic score were observed at the rotor speed stages at lower and higher levels. This is because the increase in the surface speed of the rotor increases the centrifugal force acting on the particles, so it is proportional to the square of the angular velocity of the rolls. In addition, changes in surface speed also affect the spacing of the particle bed and the time of sorting.

According to the influence of the observed magnetic field strength and the feed rate on the Fe content of the IMRS magnetic product at the horizontal stage of the feed rate center, the $\mathrm{Fe}$ content in the magnetic product increases as the magnetic field strength increases. It was also observed with changes in feed rate, the slight influence of the magnetic quality of the product. These two variables were found to have lower Fe content at lower levels. The grade value is higher at the lower feed rate and higher magnetic field strength levels. Similarly, the effect of rotor speed and feed rate on the IM content of the IMRS magnetic product at the center level of the magnetic field strength was observed. It can be seen that the Fe content of the magnetic product has a slight effect as the feed rate changes. It found that the rotor speed grade magnetic products are very sensitive to changes. Moreover, a higher grade value appears at the center of the rotor speed. This is due to the centrifugal force on the optimization of the two particles, different particles to promote flow under a given magnetic field strength.

ii. Effect of variables on the content of $\mathrm{SiO}_{2}$ in magnetic products

Similar to the Fe content, in this case, it is considered that the sorting is effective only when the silica content is the lowest. ${ }^{[14]}$ Observe the influence of the magnetic field strength and rotor speed on the $\mathrm{SiO}_{2}$ content in the magnetic product at the center of the feed rate ${ }^{[14]}$.In the center magnetic field strength and the phase of high rotor speed, low silica content was observed in the case of the magnetic product, and found a positive correlation between the ferromagnetic substance. Observing the influence of magnetic field strength and feed rate on the silica content of magnetic products at the central phase of rotor speed, it was found that the silica content of magnetic products decreased with the increase of magnetic field strength. At the same time, it was found that when the feed rate changed, the silica content did not change significantly, but the composite effect had an effect on the separation of the light gangue minerals.

From the test results, the effects of rotor speed and feed rate on the $\mathrm{SiO}_{2}$ content of the IMRS magnetic product at the center of the magnetic field strength were observed. It can be seen that as the feed rate changes at different rotor speeds, there is a slight change in the $\mathrm{SiO}_{2}$ content of the magnetic product. When the rotor speed is at the center level, the $\mathrm{SiO}_{2}$ content is at the lowest level regardless of the feed rate. This is due to the centrifugal force acting on the particles (hematite and silicate- 
containing minerals). It helps the different particles to flow at a given magnetic field strength.

iii. Effect of variables on the recovery rate of magnetic products $\mathrm{Fe}$

Similar to the above two tests, the results show the effect of magnetic field strength and rotor speed on the $\mathrm{Fe}$ recovery of the magnetic product at the center of the feed rate ${ }^{[14]}$. Higher magnetic product recovery rates are achieved at higher magnetic field strengths and lower rotor speed levels. At lower magnetic field strengths and higher rotor speed levels, the recovery is lower. This is because the lower magnetic force acting on the particles drags the particles inward, whereas if centrifugal force acts on the particles, this will cause the particles to move away from the rotor surface. This is the main reason for the poor sorting of hematite particles, which also leads to lower iron recovery .As the strength of the magnetic field increases, the recovery of iron from the magnetic product also decreases as the rotor speed of the IRMS increases. Therefore, the recovery rate of hematite particles decreases as the rotor speed increases. At the lowest magnetic field intensity the decrease in the recovery was more than the other levels of varied magnetic field intensities. This is because at lowest magnetic field intensity the magnetic force diminished and the centrifugal force gained by the particles dominated. The recovery of a hematite particle in the magnetic product decreased rapidly due to increased centrifugal force of hematite particles at higher roll speed.

Observing the influence of magnetic field strength and feed rate on the recovery of magnetic product at the center of the rotor speed, it was found that the Fe recovery rate in the magnetic product increased with the increase of the magnetic field strength. It is also found that with an increase in the feed rate, there is a decrease in the recovery at lower magnetic field intensity whereas at higher magnetic field intensity, it increases with the feed rate. Therefore, it is clear that the combined effect of feed rate and magnetic field strength on the recovery is very important. In other words, at lower magnetic field strengths and higher feed rates, the hematite particles are very poorly sorted, which in this case results in a minimum recovery. Observing the effect of rotor speed and feed rate on the magnetic product recovery rate at the central stage of the magnetic field strength, it was found that when the recovery rate is maximum, the rotor speed is lower and the feed rate is higher, and when the recovery rate is the smallest, the feed rate and rotor speed is at a high level. As the layer of particles moving over the rotor increases, the top layer particles pass through the low magnetic gradient region above the rotor; thus illegitimate separation occurs. Therefore, as the feed rate increases, the recovery of hematite particles in the magnetic portion increases.

iv.Effect of particle trajectory

In another document ${ }^{[5]}$, it is known that the position and particle size of the magnetic separator are important parameters for sorting the granular magnetic and nonmagnetic products. Particle trajectory mainly controlled by the magnetic force of gravity, and centrifugal force acting on the particles. Regarding the position of the splitter of the induction roll magnetic separator, it can be defined as the angle at which the particles leave the rotor surface and contact the top line of the splitter .As a result of observing the change in the position of the magnetic separator by the literature data, as the position angle of the magnetic separator decreases, the particles move toward the center of the rotor. The yield of the magnetic product drops sharply as the angle of the magnetic separator position increases. It is also found that the magnetic product has the smallest iron content when the magnetic separator has the smallest angular position. Similarly, the sorting efficiency is highest when the magnetic separator has the smallest angular position.

Reduce product quality is due to the attractiveness of the particle interlocking magnetic flow. In general, various product bins are used to observe particle trajectories of different masses (by combining particle size and density) and magnetic susceptibility particle trajectories by means of a manifold, and the position of the magnetic separator is an important variable in the induction roll magnetic separator. As can be seen from Figure 2, the trajectory of the particles to the product bin is determined based on the force acting on the particles in a given magnetic field. In addition, the effects of particle characteristics and particle trajectories of different minerals are discussed in published literature ${ }^{[3]}$ [4] [5] [6].

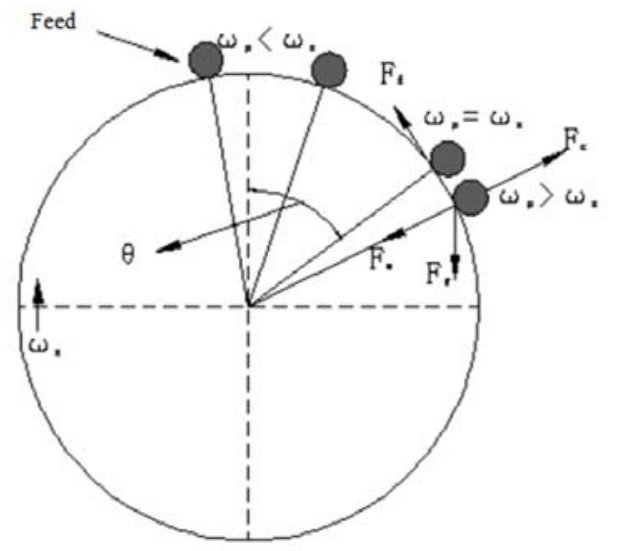

Figure. 2. Particle motion and trajectory phase during magnetic scrolling.

\section{v.Particle size effect}

The test on the influence of particle size is known from the literature ${ }^{[3]}$.Two sets of experiments with different particle sizes were designed to ensure that the other variables were constant. By observing the rotor speed of the IRMS, IRMS was found to be very sensitive to particle separation. Other parameters such as magnetic field strength and feed rate are maintained at a constant value, respectively. It can be seen that the yield of the magnetic product under the finer size material is greatly reduced as the rotor speed increases relative to the coarser particle size material. This change may be due to the yield of the higher centrifugal force of the coarser particles, so that the dominant role in the material particles together as a non-magnetic product separation. Therefore, for feeds of coarser size, the separation efficiency decreases as the rotor speed increases, while the effect on the finer size feed is reversed. 


\subsubsection{Application}

It can be seen from the above that the induction roller type magnetic separator is more effective in processing particles of a relatively coarse size. In the treatment of fine-grained materials, due to the lesser amount of rotor treatment, coupled with the influence of air flow, particle-particle adhesion and particle-rotor adhesion, the separation effect of the magnetic separator on fine materials is further affected. IRMS is widely used in the beneficiation of beach sands and the removal of weak magnetic impurities from glass sand, andalusite, feldspar, wollastonite, magnesite and other industrial minerals. The magnetic separator can also be used for the concentration of minerals such as chromite, monazite and wolframite.

\subsection{Cross-belt magnetic separator}

Cross-belt magnetic separator is used to concentrate the one paramagnetic mineral earliest type of separator. Figure 3 shows a schematic of such a magnetic separator. The dry material is conveyed onto the conveyor belt in a single layer and transferred between the magnetic poles of the magnetic system. The conveyor belt is transferred between the poles of two or more electromagnets. The lower support is flat and immovable, and the corresponding upper support can be raised or lowered depending on material properties. In the upper part of the magnetic separator is a cross-belt for distinguishing between magnetic material and non-magnetic material. The cross-belt spans the upper support member and runs around a series of pulleys and runs perpendicularly to the conveyor belt. As the material passes, the magnetic particles in the material are attracted to the cross-belt to the front side and exit into the collection trough. An advantage of CBMS is the recovery of several magnetic products of different magnetic susceptibility in each feed. However, the low throughput of the device is a major drawback, making it unpopular in the high-volume mineral industry.

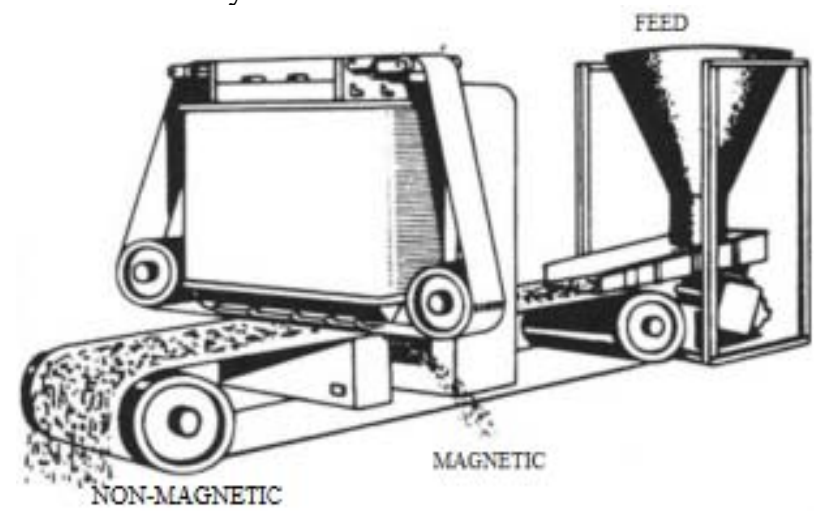

Figure.3. Schematic diagram of particle separation in a crossbelt magnetic separator

\subsubsection{Effect of Variables on Particle Separation}

The main process variables of the separator are magnetic field induction on the belt surface, coil current, pole gap, feed rate, main belt speed, cross belt thickness and speed.
i.The influence of magnetic field strength

Magnetic field strength is one of the important parameters affecting the sorting effect. In the test, the magnetic field induction intensity was adjusted by adjusting the current on the coil .In the test of separation of titanium dioxide and iron oxide from Egyptian albite samples, the effect of magnetic field strength was studied at a constant feed rate of CBMS ${ }^{[7]}$. The main purpose of this study was to remove harmful iron gangue minerals. It can be explained that when the magnetic field strength rises to a certain value, the increase in the rejection of these iron-containing impurities is then kept constant. From this, it can be found that as the strength of the magnetic field increases, the removal rate of iron minerals decreases, and the product grade increases.

ii. Influence of belt speed and feed size

The belt speed of CBMS is an important parameter that determines the residence time of a particle in a given magnetic field. The effects of belt speed, feed size and applied magnetic field were investigated by experiments in which iron-containing minerals were separated from nepheline-syenite samples ${ }^{[8]}$. It was found that as the belt speed of the CBMS increased, the iron content of the non-magnetic product also increased. Feed rate is another parameter that affects the separation of CBMS particles. The change in feed rate results in the formation of a layer of particles on the surface of the belt. As the thickness of the feed layer on the surface of the CBMS tape rises, the sorting process deteriorates due to the shielding effect. This has a significant effect on the quality of the concentrate and the capacity of the magnetic separator. In addition, efficient separation of materials is facilitated when the feed rate is low. Similar observations are also evident in the literature to observe the separation of iron-bearing minerals from albite samples ${ }^{[7]}$. The effect of the feed rate was studied at a specific magnetic field strength and it was found that the removal rate of the coloring material decreased as the feed rate increased. It can be observed that the feed rate is inversely proportional to the removal rate of iron oxide and titanium dioxide. At lower feed rates, the removal rates of iron oxide and titanium dioxide are rather high. This explains that at low feed rates, there is only one layer of particles on the belt, which provides an opportunity for strong electromagnets to absorb coloring materials and improve separation performance.

However, the belt speed of the particle sorting effect needs further study. The combined effects of different variables on the belt surface are not mentioned in the literature. The sorting of particles in the system must be mathematically understood and defined by considering the physical properties of the mineral to be sorted.

\subsubsection{Application}

In most cases, CBMS is used for sorting cleaning stage, i.e., removal of harmful magnetic particles and paramagnetic particles from the bulk material. It is worth noting that its application to minerals other than sand is very limited. 


\subsection{Lifting Roller Magnetic Separator}

There is also a magnetic separator called a lift roller magnetic separator, and the lift roller magnetic separator is also operated under the principle of an electromagnetic magnetic separator. The material is fed in a slightly inclined manner on the side of the roll. The magnetic particles are attracted to the magnetic induction roller, and the non-magnetic particles that are not affected by the magnetic field are lowered by gravity. When the magnetic particles lose the magnetic field in this case, they also fall due to gravity. The particle separation in the lift roll magnetic separator is shown in Figure. 4. Among them are dry and free-flowing particles, which will be used in granular and powdery materials. When the machine is non-magnetic, the magnetic particles are removed from the fluid by gravity, thereby minimizing the interception of particles by the magnetic separator. Various types of magnetic separators produce a new magnetic product. The working principle of the grounding roller is fixed between the two magnetic blocks. The magnetic flux generated from the bottom of the roller is upward. The roller has a trough surface with a convergent magnetic field and produces a magnetic field strength of up to $2 \mathrm{~T}$. By using this mechanism of LRMS, paramagnetic particles can be lifted and collected as magnetic parts. The lift magnetic separator is more selective than the induction roller magnetic separator. However, the main limitation of the lift magnetic separator is its low capacity. This magnetic separator is used to separate garnets from monazite and rutile in the heavy mineral industry.

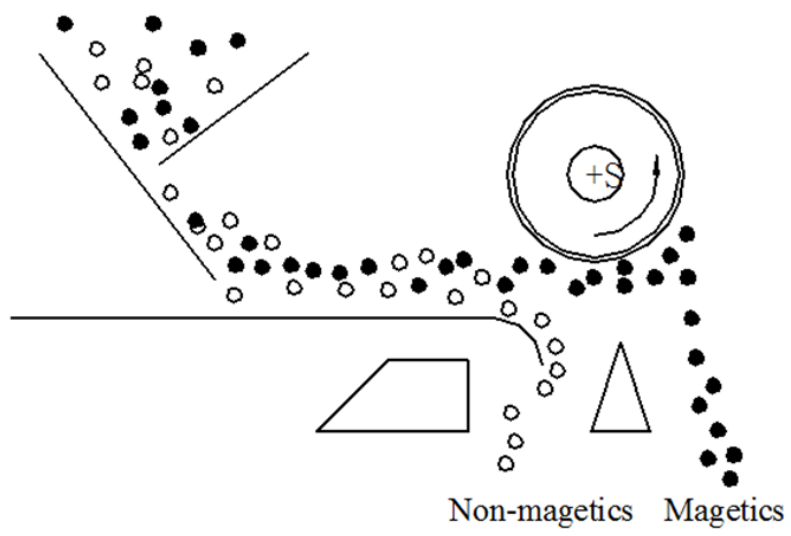

Figure 4 Particle separation in lifting roller magnetic separator

\section{Permanent Magnet Magnetic Separator}

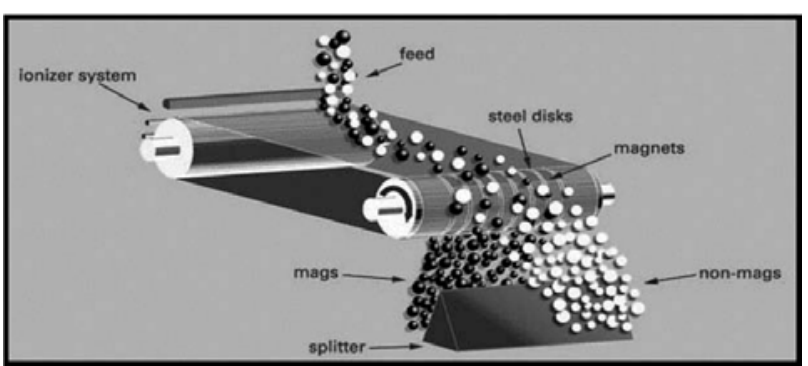

Figure 5 Rare Earth Roller Magnetic Separator Particle Separation

Although the concept of permanent magnet roller separators dates back to 1968 (Andreachi and Palasvirta, 1965).However, the permanent magnet by a permanent magnet separator plate of low carbon steel or intersecting rings, but with the emergence of rare earth permanent magnet material was contributed to its appearance. Permanent magnet roller magnetic separators were originally made of samarium cobalt as a permanent magnet, which is commercially available, but they were quickly replaced by neodymium iron boron magnets. The movable portion is a roller separator by a disk or a permanent magnet rings, interposed between the lowcarbon steel rings. Figure 5 schematically shows a permanent magnet generating a magnetic field in the magnetic separator roll. To make it easier to remove the magnetic particles, the belt covering the rolls can be made as thin as possible to keep the feed material as close as possible to the magnet. Belt supported by idler rollers. Beneath the conveyor to collect material discharge hopper, adjustable flow diverter different material is transferred to the hopper of the collection box. The permanent magnetic roll separator can be designed as a single strand of multi-stage separation, the case of discs with a permanent magnet, the adjacent permanent magnet ring disposed opposite to each other with the same polarity. With this design, the intensity of the magnetic field gradient produced is much larger than that of the conventional induction roller magnetic separator. With this design, the intensity of the magnetic field gradient produced is much larger than that of the conventional induction roller magnetic separator. The samarium cobalt permanent magnet prepared from (rare earth + cobalt alloy) alloys, and as the roll diameter increases, the residence time of the particles in a magnetic field is increased. The same permanent magnetic force generated by a magnetic roller and the induction magnetic roller. Compared to induction roller magnetic separators, permanent roller magnetic separators have many advantages, such as low energy consumption, low instrument quality, and large footprint. The permanent magnet roller magnetic separator can process particles of about $25 \mathrm{~mm}$. But it also has some shortcomings including belt wear and uncontrollable magnetic field strength. Although permanent magnet roller magnetic separators are mainly dry machines, they can also be used as wet magnetic separators at certain times, for example, in the concentration of diamonds. 


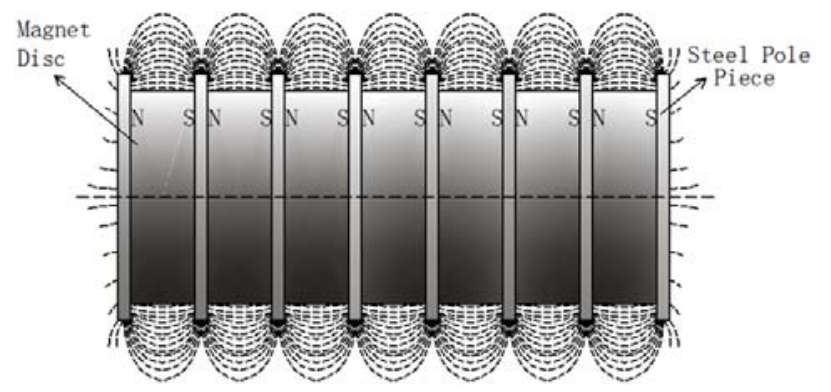

Figure 6 Magnetic field generated on a roller magnetic separator

\subsection{Rare Earth Roller Magnetic Separator}

RERMS is a permanent high intensity magnetic separator, mainly paramagnetic mineral dry sorting machine having the higher operating costs. With the advent of the rare earth permanent magnet material roll magnetic separator, we have been successfully configured to use larger magnetic roll separator electromagnetic alternating laminations of magnetic and non-magnetic ${ }^{[13]}$. The RERMS feed material is introduced onto a magnetic roller with a ribbon, and the magnetic particles are attracted by the magnetic roller. The non-magnetic particles are rejected by centrifugal force, gravity and other forces due to lack of magnetism [9]. A schematic of particle separation in RERMS is shown in Figure 6. Rare earth permanent magnet improved availability and affordability further extends the applicability of the sorter drum moderate or even weakly magnetic material. Moreover, when the material enters the magnetic zone, no material rebounds or scatters under the action of the magnetic force, and all materials enter the magnetic region at the same horizontal velocity. All of this helps to separate the magnetic material from the non-magnetic material. Moreover, the use of rare earth permanent magnets can greatly reduce the quality and size of the induction roller magnetic separator.

Because of the excellent separation efficiency of RERMS, they replace the traditional electromagnetic high-strength magnetic separator. Roller Magnetic capacity depends on the diameter of the roller. Diameter of the roller magnetic separator is determined according to the particle size to be treated ${ }^{[10]}$. Therefore, the roller magnetic separator is more efficient and can be adjusted more easily according to needs. It is not only effective and efficient means of conventional particle size, but also particularly effective for finer particles $(<75 \mu \mathrm{m})$ work material . In addition, samples from Turkey feldspar are processed to sorting colored impurities ${ }^{[11]}$ in the RERMS. Non-magnetic parts composed of feldspar and quartz and minimal titanium and iron oxide were found to be suitable for the ceramic industry.

\subsubsection{Effect of Variables on Particle Separation}

The parameters affecting the RERMS sorting process can be divided into three categories: design variables, process variables, and feed characteristics. The results show that the diameter of the roller, the magnetic field strength of the roller, the design type and magnetic steel ratio, the type and thickness of the belt, and the sorting grade are all key design parameters in RERMS. In addition, it was found to control the separation process variables is critical to achieving optimal performance on grade and recovery. Therefore, the feed rate, magnetic separator position, feed rate, and feed depth on the RERMS belt are key parameters for controlling separation efficiency. In addition, particle size, density, shape, and distribution feed susceptibility characteristics in the design and optimization process is critical.

i. Effect of roll speed, feed rate and split position

Roller speed and feed rate are important parameters that are often adjusted based on changes in industrial feed characteristics. Separation of impurities in ironcontaining perlite was studied by a permanent magnet roller magnetic separator. It was found that $\mathrm{Fe}_{2} \mathrm{O}_{3}$ content and particle size varied at different separation stages of different roll speeds. By changing important variables such as roll speed and feed rate, it is possible to optimize the production of high quality glass products. In some literature has appeared similar test, the powder was observed in the separation of a fluid catalytic cracking $\left(\mathrm{F}_{\mathrm{CC}}\right)$ in the belt to affect the type and thickness of the belt ${ }^{[12]}$. There is another study based on the separation of hematite from silicate-containing gangue minerals .The influence of the interaction between the roll speed, the feed rate and the split position of RERMS on the low-grade ore hematite fine powder was studied from the slope of the three-dimensional surface map. As a result, it was found that the roll speed and the position of the magnetic separator have a more significant influence than the feed rate. The analysis was performed from the slope of the three-dimensional surface map. It was found that the grade of the magnetic product increased as the roll speed increased, and the intermediate level was also reflected as a higher value of the separation efficiency. However, the roll speed is further increased beyond the peak, resulting in decreased quality. This change in mass may be due to the fact that the increase in roll speed makes the net resultant force on the hematite particles not optimal. It is clear that the RERMS roll speed determines the separation of particles on a given magnetic roll. Similarly, the interaction can be observed along with other variables and recovery results.

ii.Particle size effect

Particle size is an important parameter for magnetic selection, which determines the other design of the magnetic separator and the corresponding adjustment of the process parameters during the separation process. Ibrahim et al. studied the effect of magnetic separator position and roll speed on particle separation of different particle size components. It has been concluded that the closed particle size range has better efficiency in this type of magnetic separator compared to a wider particle size range feed. Many authors have analyzed the effect of particle size on RERMS separation ${ }^{[13][3]}$. The effect of 
particle size on a given magnetic field is well analyzed for particle trajectories and product mismatch (Trpices et al., 2017).Figure 7 analyzes the effect of feed size on the efficiency of a particle size based RER magnetic separator in an interesting manner. The particle trajectories of these non-magnetic particles are determined only by the centrifugal force. When the nonmagnetic particles and allowed to drop unhindered movement in the roll, substantially according to their particle size the particle size decreases as shown in FIG. 7. The large particles will move further from the smaller centerline of the roll. Thus, usually lower than the surface velocity of small particles to large particles treated. When a magnetic roller is used, magnetic particles having a stronger magnetic field are usually pinned on the surface of the roller until they are released from the magnetic field, and magnetic particles having a weak magnetic field can only be deflected by the magnetic field, causing them to deviate from the normal path. When this happens, overlap occurs in the largersized weak magnetic particles and the small nonmagnetic particles ${ }^{[18]}$.If a product separator is provided to eliminate the large size weak magnetic particles, many small non-magnetic particles will be incorporated into the magnetic product. This is due to the entrainment of the magnetic portion by the non-magnetic fine particle fraction. On the other hand, when the product magnetic separator is set to recover non-magnetic particles of a relatively small size, the non-magnetic product after sorting will contain many large-sized weak magnetic particles. This problem can generally be divided into closed magnetic separation after sorting by particle size and components to overcome the feedstock. In addition, these components can be separated in multiple stages to track, to enhance the recovery.

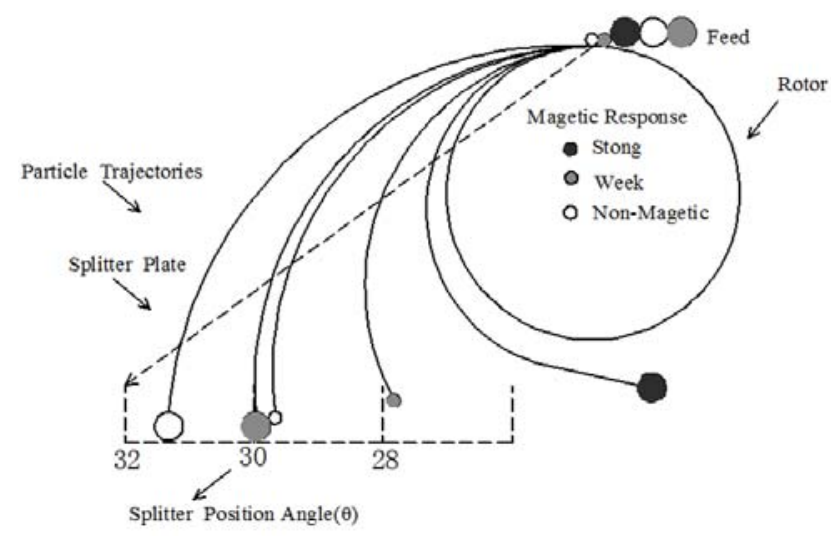

Figure 7 Particle trajectories for different particle size and magnetic susceptibility

\subsubsection{Application}

The magnetic separator is also widely used in different mineral industries for the preconcentration and cleaning of minerals. It is also used as a separator for the removal of iron oxide from glass, ceramics, abrasives, chemicals, bones and calcined magnesite and spray dried ceramic pellets. It can also be used in heavy mineral sands industry rutile and zircon cleaning. It is also for recovering metals from scrap steel in secondary processing. Few applications are focused on the microscopic fractionation of coal, and the reduction of the sulfur from the coal.

\subsection{Rare Earth Drum Magnetic Separator}

\subsubsection{Brief Description}

The rare earth drum magnetic separator is the most commonly used low-strength device for sorting strong and medium magnetic particles. As shown in Figure 8, the device consists of a rotating non-magnetic drum containing a plurality of fixed magnets of alternating polarity, the electromagnets typically being made of permanent magnets. The sorting material is fed from the top of the surface of the drum, and the non-magnetic portion leaves the drum by centrifugation, and the magnetic portion is retained by the magnetic field on the surface, and then falls outside the magnetic field, thereby being deflected. In addition to magnetic and nonmagnetic products, intermediate products are obtained based on material properties and interlocking characteristics. In the present study, during the operation of the rare earth drum, ilmenite was recovered in the magnetic portion, and the garnet included rutile, zircon, and sillimanite in the non-magnetic portion in the middle portion and other heavy minerals. In this test, a certain variable was changed, and the remaining variables were kept constant. Observed the results of the test data, it was found that most of the test data showed a peak factor.

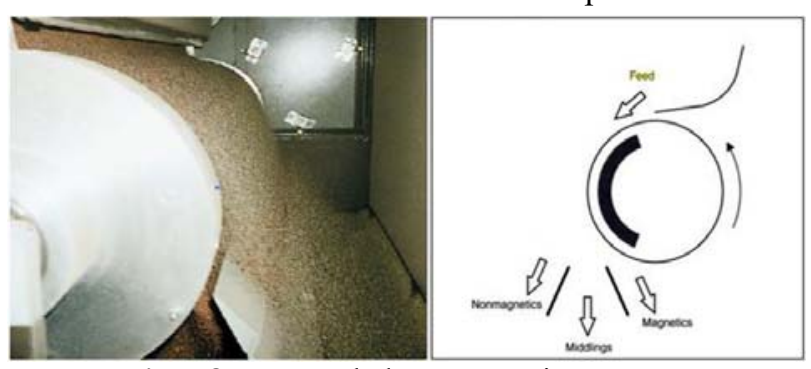

Figue 8 Rare earth drum magnetic separator

\subsubsection{The Influence of Variables}

\section{i.The effect of feed rate}

It is observed that with the increase of feed rate, the content of ilmenite in the product increases gradually, and then increases to the peak value, and the content of ilmenite begins to decrease gradually. At low feed rates, the finer feed will be affected by the high magnetic susceptibility of the drum. Therefore, part of the ilmenite and garnet particles (slightly magnetic) are sorted in the intermediate product portion, so that the quality of the ilmenite product is reduced, resulting in low recovery. It can be seen that as the feed rate increases, the recovery and grade of ilmenite exceeds the peak. As the feed rate is further increased, the feedstock has an overload effect, the separation process is ineffective, and finally the grade and recovery rate are reduced.

ii. The impact of the drum speed 
When the test is run, the other parameters of the magnetic separator are maintained at a constant critical value. Observing the data of the drum speed in a certain section, as the rotation speed of the drum increases, the recovery rate and grade of ilmenite gradually increase, and with the drum speed rise again, the recovery rate and grade of ilmenite gradually decrease.

At low speed of the drum, this time the magnetic medium and high magnetic garnet ilmenite feedstock deposited on the drum is discharged to the magnetic portion, thus resulting in a lower grade and recovery of ilmenite. As the drum speed increases, the high-magnetic ilmenite and medium-magnetic garnet begin to differentiate due to their own magnetic field, so the ilmenite grade and recovery rate increase. When the drum rotation speed increases after the peak, the particle sorting efficiency does not rise at this time. Mainly fine garnet particles are sorted together with ilmenite, which in turn leads to a decrease in ilmenite grade and recovery.

iii. Effect of feedstock vibration rate

When the magnetic separator feeds the vibration value up, the other parameters of the magnetic separator are maintained at a constant critical value. The recovery and grade of ilmenite increased with increasing feed vibration rate, but decreased with increasing feed vibration velocity after exceeding the peak. When the feed vibration rate is low, the grade and recovery of ilmenite is low. It was observed that the ilmenite was not effectively sorted due to insufficient feed to the drum, resulting in partial ilmenite in the middle and nonmagnetic portions of the sorting result.

As the material to increase the rate of vibration, can be made on the separator drum to a uniform flow of feed, product grade and recovery of ilmenite also will increase .As the feed vibration rate increases beyond the peak, the ilmenite grade and recovery are gradually reduced due to the unstable feed rate of the separation drum.

iv.The influence of feed temperature

The test while keeping the other parameters of the magnetic separator is constant. As the feed temperature increases, the recovery and grade of ilmenite increases to a peak, and then the ilmenite recovery and grade increase gradually decrease as the feed temperature increases.

At lower feed temperatures, the separation of ilmenite was observed and it was found that the particles could not be effectively sorted due to the influence of humidity, resulting in low grade of ilmenite and low recovery .Therefore, the feedstock is kept at the lowest temperature, and as the feed temperature increases, the sorting starts to be effective, and the grade and recovery of ilmenite begins to rise. As the feed temperature is further increased, the feedstock is refined and the particles segregate, resulting in unstable feed flow conditions and unfavorable separation processes on the magnetic separator. The loss of ilmenite was observed in the garnet and non-magnetic parts, rather than in the magnetic part. As a result, the grade and recovery of the ilmenite product are reduced, thus creating unstable feed stream conditions and unfavorable separation processes.

v.Effect of splitter position
The grade and recovery rate of ilmenite varies with the sorting position of the magnetic separator. At the lower position of the magnetic separator, a large amount of non-magnetic material is sorted at this time, and finally the ilmenite product appears in the non-magnetic section together with the garnet, sillimanite, zircon and the middle portion. This significantly reduces the quality and recovery of magnetic domain ilmenite.

When the position of the indexer increases to the peak value, the magnetic segmentation magnetic separator cutting will be in the proper position of the maximum mass of the sorted magnetic material (ilmenite product), resulting in high recovery of ilmenite.

As the grader position increases beyond the peak, the garnet and ilmenite are sorted in the magnetic portion, resulting in a reduction in ilmenite recovery and grade.

\subsubsection{Application}

Rare earth magnetic separator drum are used for fine feed for dry magnetic separation processes. In most dry process concentrators, in the early stages of the dry separation process, two or more paramagnetic minerals are typically separated into individual products using a rare earth drum magnetic separator (RED). According to the literature, compared with the rare earth roller magnetic separator, the drum magnetic separator (RED) and the fine and superfine material sorting effect is better. In mineral separation plant, typically magnetic roll separator for recovering high-value products and final cleaning, such as rutile and zircon, suitable separation if required, higher field strength is required ${ }^{[15]}$. For high susceptibility mineral ilmenite, and the like of rare earth magnetic separator drum magnetic separator suitable for the primary intensity magnetic separation.

\section{Conclusion}

The magnetic separators discussed in the above literature have a wide range of industrial applications and have extremely important applications in the mineral industry. New applications and design concepts in these magnetic separators have been developed. Although China has made significant research and development in the field of iron ore magnetic separation, the resources it has developed and utilized are already at the forefront of the world .However, due to the complex distribution of resources in China. Not only that, but China's overcapacity is still present. In terms of infrastructure, there are still gaps between China and those big countries. Reducing the energy consumption of the magnetic separator and making it large, specialized and energy-saving is still the key development of the magnetic separator. China still lacks high technology and still needs to develop cautiously.

\section{References}

1. D.V.S. Rao, Crc Pr Inc, Belkema(2011) 
2. W. A. P .J. Premaratne, N.A. Rowson, Phys. Sep. Sci. Eng. 12(2003), 215-222.

3. S. K. Tripathy, P. K. Banerjee, N.Suresh,Int.J.Min.Met Mater, 22(2015), 661

4. V. Singh, S. Nag, S. K. Tripathy, Powder.Technol, 244(2013), 85-92.

5. S. K. Tripathy, V. Singh, Y. R. Murthy, P. K. Banerjee, N. Suresh, Int.J.Miner.Process, 160(2017), 16-31.

6. S. K. Tripathy, Y. R. Murthy, V. Singh, N. Suresh, Miner.Process. Extr M, 37(2016),196-210.

7. F. H. El-Rehiem, A. El-Rahman, Min.Proc.Extmet. Rev, 117(2008), 171-174.

8. S. S. Ibrahim, H. A. Mohamed, T.R.Boulos, Physicochem Probl Mi, 36, 173-183.

9. D. A. Norrgran, J. A. Marin, Miner.Metall. Proc, 11(1994), 41-45.

10. J. Svoboda, T. Fujita, Miner Eng, 16(2003), 785792.

11. O. Y. Gülsoy, E. C. Orhan, Physicochem Probl Mi, 38(2004), 301-309.

12. T. L. Goolsby, H. F. Moore, Sep Sci Technol, 32(1997), 655-668.

13. S. S. Ibrahim,M. M. Farahat, T. R. Boulos. Particul Sci Technol, 35(2017), 21-28.

14. S. K. Tripathy, P. K. Banerjee, N. Suresh, Y. R. Murthy,V. Singh, , Miner Process Extr M 38(2017),339-365

15. Arvidson, B.Arvidson ,D.Zhu, (2007). Outotec Oyj. US Patent 7296687. 